Acta Crystallographica Section B

Structural

Science

ISSN 0108-7681

Editor: Sander van Smaalen

\title{
Structure of barium chlorapatite
}

M. Hata, F. Marumo, S. Iwai and H. Aoki

This electronic document was scanned from an archival copy of material deposited to accompany a paper published in an IUCr journal. In many cases the only accessible copy was a microfilm of a poor-quality original. 


\section{ond

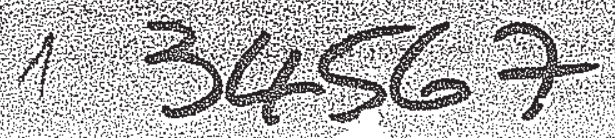

Tre antsotroplc thental parameters for all atoms $(x, 104)$

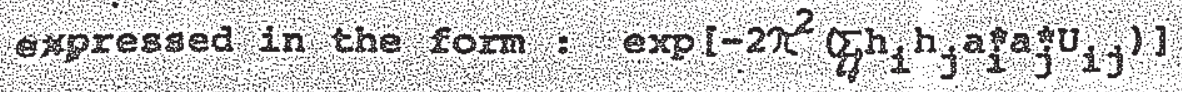

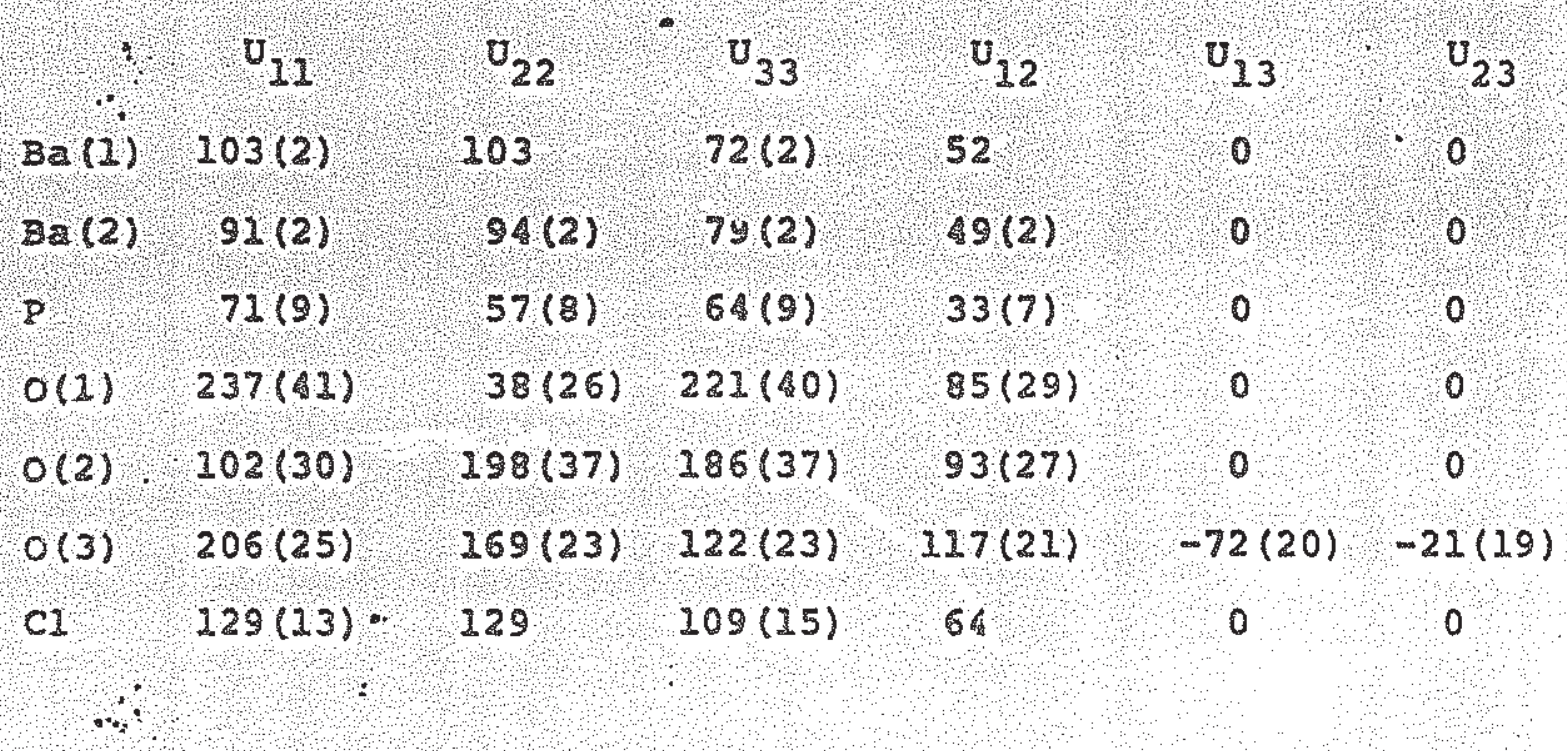


DASERVED AND CALCULATED STRUCTURE FACTORS $(\times 100)$

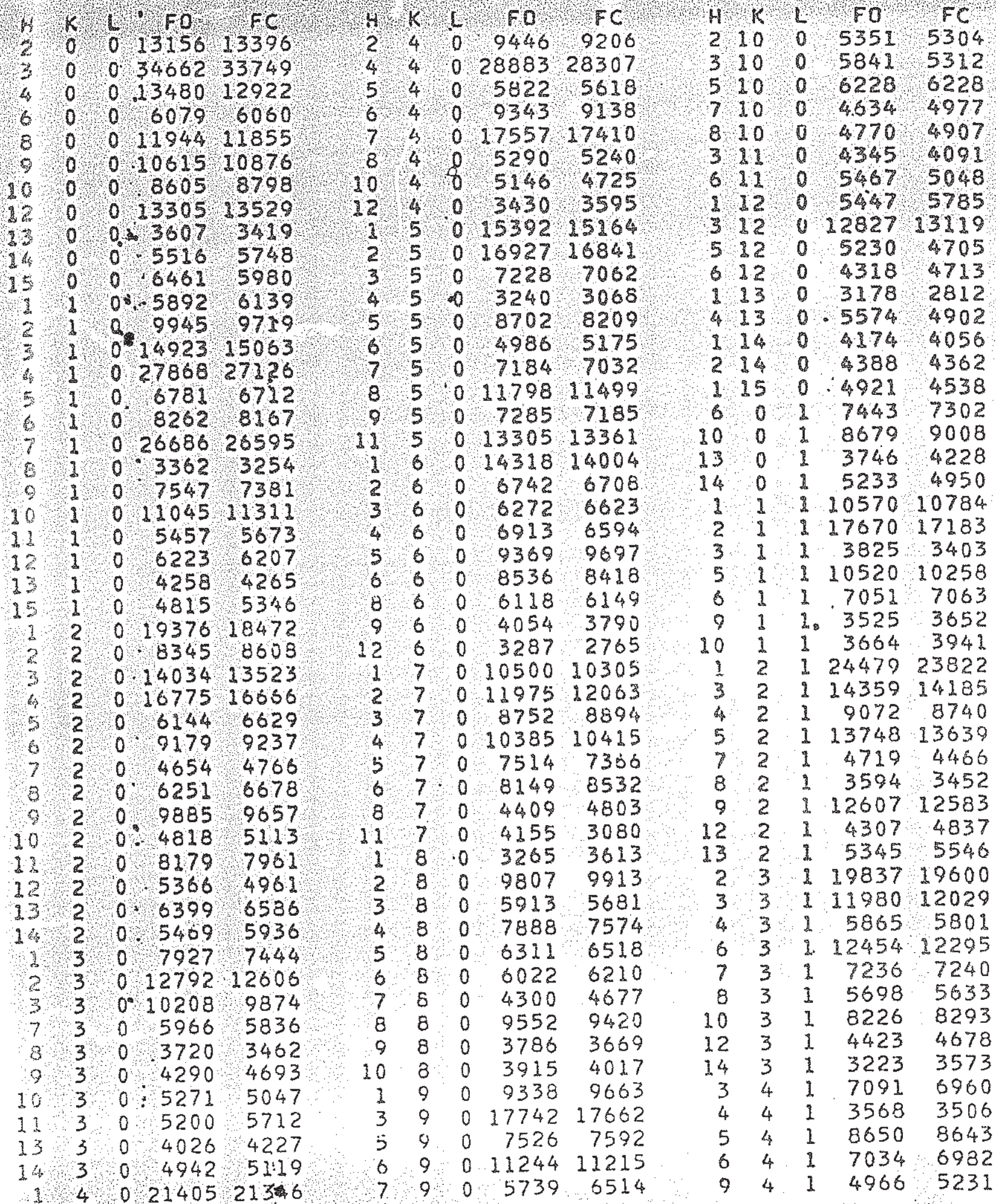




\section{OBSERVED AND CALCULATED STRUCTURE FACTORS $(\times 100)$}

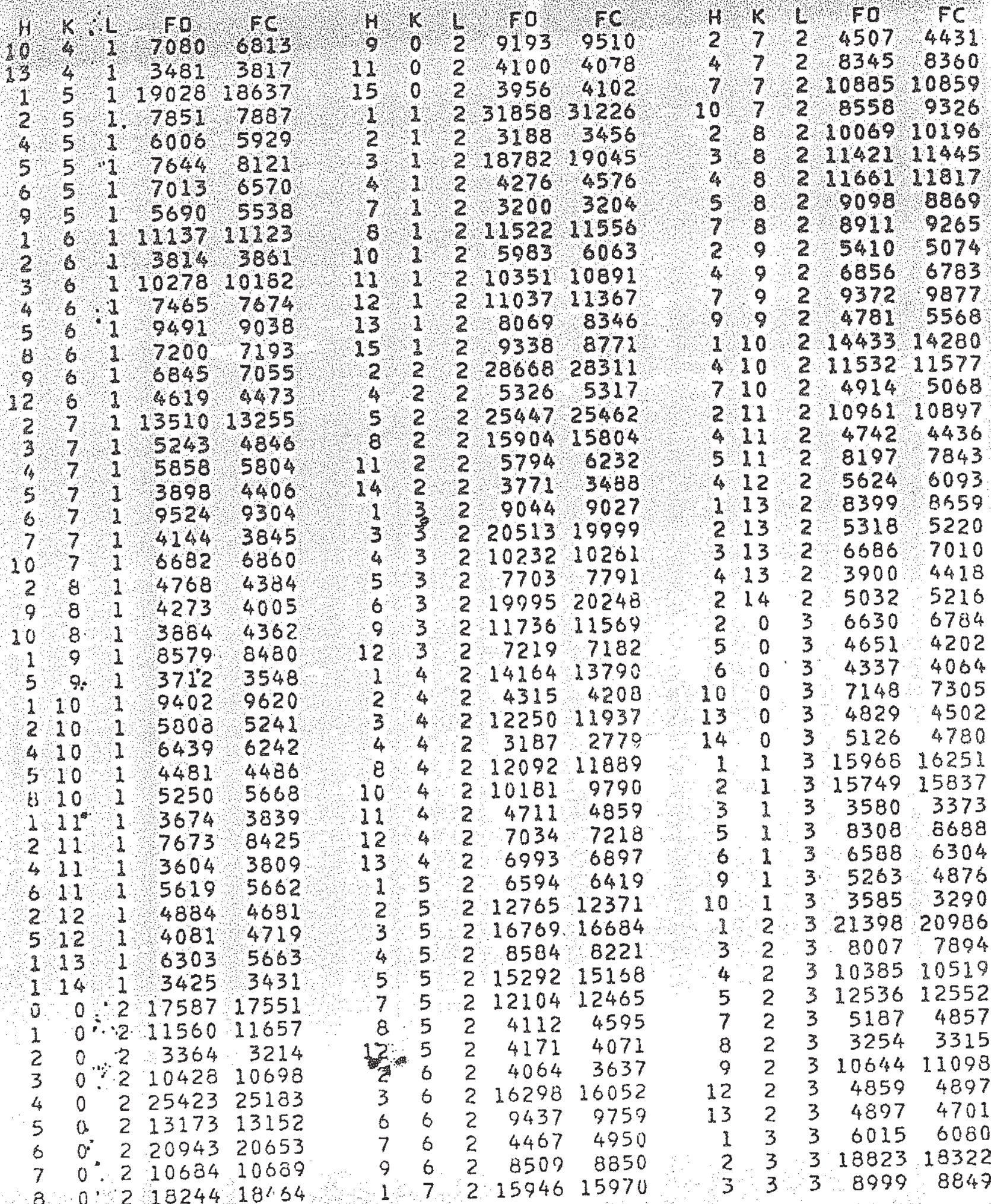


OBSERVED AND CALCULATED STRUCTURE FACTORS. $(1100)$

\begin{tabular}{|c|c|c|c|c|c|c|c|c|c|c|c|c|}
\hline $\mathrm{K}$ & 10 & FC & A. & $\mathrm{K}$ & $L$ & $\angle F O$ & $F C$ & 4 & $k$ & L & $\mathrm{Fo}$ & FC \\
\hline 3 & 8813 & 8830 & 2 & 14 & 3 & 4135 & 3499 & 5 & 4 & 4 & 5306 & 5118 \\
\hline & 6372 & 6399 & 0 & & 4 & 44647 & 47806 & 6 & 4 & 4 & $\begin{array}{r}7550 \\
\end{array}$ & $\begin{array}{r}7318 \\
\end{array}$ \\
\hline 3 & 5256 & 5435 & 2 & 0 & 4 & 8511 & 8582 & 7 & 4 & 4 & 15877 & 16333 \\
\hline 3 & 7879 & 7818 & 3 & 0 & 4 & 22930 & 23444 & 8 & 4. & 4 & 55302 & 5562 \\
\hline 3 & 3903 & 3577 & 4 & 0 & 4 & 11700 & 11815 & 1 & 8 & 4 & 13919 & 14577 \\
\hline 4 & 8559 & 8568 & 6 & 0 & 4 & 6685 & 6785 & 2 & 5 & 4 & 13139 & 13332 \\
\hline 4 & 6889 & 6961 & 8 & 0 & 4 & 9477 & 9615 & 3 & 5 & 4 & 7334 & 7263 \\
\hline 4 & 7326 & 7349 & 9 & 0 & 4 & 10427 & 10129 & 5 & & 4 & 5149 & \\
\hline 4 & 4115 & 4.99 & 10 & 0 & 4 & 7217 & 7442 & 6 & 5 & 4 & 3859 & 370 \\
\hline 4 & 6342 & 6585 & 12 & 0 & 4 & 11499 & 11803 & 7 & 5 & 4 & 6187 & 6121 \\
\hline 4 & 3292 & 3667 & 14 & 0 & 4 & 5431 & 5860 & 8 & 5 & 4 & 9931 & 20294 \\
\hline 5 & 16549 & 16367 & 15 & 0 & 4 & 5748 & 5343 & 9 & 5 & 4 & 6547 & 84 \\
\hline 5 & 8833 & 8563 & 1 & 1 & 4 & 7912 & 8203 & 11 & 5 & 4 & 12820 & 12806 \\
\hline 5 & 4781 & 5434 & 2 & 1 & 4 & 12459 & 12657 & 1 & 6 & 4 & 10381 & 10445 \\
\hline 5 & 7114 & 7309 & 3 & 1 & 4 & 5301 & 4998 & 2 & & 4 & 4203 & 4358 \\
\hline 5 & 5240 & 4888 & 4 & 1 & 4 & 24033 & 24396 & 3 & 6 & 4 & 6642 & 6631 \\
\hline 5 & 4182 & 4209 & 5 & 1 & 4 & 7975 & 7585 & 4 & 6 & 4 & 6335 & 6667 \\
\hline 6 & 8348 & 8376 & 6 & 1 & 4 & 8716 & 8791 & 5 & & 4 & & 7979 \\
\hline 6 & 8959 & 878 & 7 & 1 & 4 & 24791 & 24877 & 6 & 6 & 4 & 7217 & 7340 \\
\hline 6 & 7188 & 7110 & 8 & 1 & 4 & 4320 & 3976 & 8 & 6 & 4 & 5841 & 5763 \\
\hline 6 & 990 & 9900 & 9 & 2 & 4 & 6047 & 5805 & 1 & & 4 & 7584 & 7628 \\
\hline 6 & 66 & 638 & $\begin{array}{l}10 \\
11\end{array}$ & 1 & 4 & 10298 & 10422 & 2 & & 4 & 11014 & 11106 \\
\hline 7 & 3906 & $\begin{array}{r}6175 \\
13738\end{array}$ & 12 & 1 & 4 & $\begin{array}{l}5186 \\
0203\end{array}$ & $\begin{array}{l}5201 \\
6248\end{array}$ & 3 & 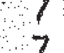 & & $\begin{array}{r}6655 \\
10412\end{array}$ & $\begin{array}{r}6589 \\
90067\end{array}$ \\
\hline 7 & 6864 & 701 & 13 & 1 & 4 & 3752 & 3473 & 5 & 7 & 4 & & $\begin{array}{l}1006 \% \\
5800\end{array}$ \\
\hline 7 & 5707 & 5609 & 1 & 2 & 4 & 12611 & 12547 & 6 & 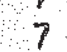 & 4 & 77 & 7657 \\
\hline 7 & 3313 & 2945 & 2 & 2 & 4 & 68 & & 9 & 7 & 4 & 40 & 3851 \\
\hline 7 & 9205 & 941 & 3 & 2 & 4 & 8139 & 8422 & 1 & 8 & 4 & 5503 & 5732 \\
\hline 7 & 3331 & 327 & 4 & 2 & 4 & 13233 & 13411 & 2 & 8 & 4 & 9482 & 9207 \\
\hline 7 & 6601 & 6584 & 6 & 2 & 4 & 8096 & 83 & 3 & 8 & 4 & 44 & 4.773 \\
\hline 8 & 4240 & 41 & 7 & 2 & 4. & 375 & & & 8 & 4 & 5686 & 5448 \\
\hline 8 & 3 & 40 & 8 & 2 & 4 & 4337 & 3990 & 5 & 8 & 4 & 6749 & 6303 \\
\hline 8 & 3384 & 3108 & 9 & 2 & 4 & 8029 & 8832 & 6 & 8 & 4 & & 4987 \\
\hline$B$ & 4280 & 404 & 10 & 2 & 4 & 4029 & 37 & 7 & 8 & $8^{4}$ & 4458 & 4555 \\
\hline 9 & 9585 & & 11 & 2 & 4 & 7650 & 7569 & 8 & $B$ & 4 & 8507 & 9088 \\
\hline 9 & 3716 & 358 & 12 & 2 & 4 & 4184 & 419 & 1 & 9 & 4 & 7948 & 7965 \\
\hline 9 & 3500 & 345 & 13 & 2 & 4 & 5627 & & 3 & 9 & 4 & 24896 & 24711 \\
\hline 9 & 3823 & 336 & 14 & 2 & 4 & 6541 & 5910 & 5 & 9 & 4 & & 6579 \\
\hline 10 & 9759 & 97 & 2 & 3 & 4 & 9467 & 906 & 6 & 9 & 4 & 9325 & 9254 \\
\hline 10 & 6153 & 559 & 3 & 3 & 4 & 9140 & 93 & 7 & 9 & 4 & 6160 & 5414 \\
\hline 10 & 7477 & 721 & 7 & 3 & 4 & 5899 & 0244 & 2 & 10 & 4 & 3576 & 4052 \\
\hline 10 & 4814 & 478 & 8 & 3 & 4 & 4291 & 4302 & 3 & 10 & 4 & 4397 & 4373 \\
\hline 10 & 5570 & 5597 & 9 & 3 & 4 & 4929 & 4865 & 5 & 10 & 4 & 6157 & 5710 \\
\hline 11 & 3882 & 410 & 10 & 3 & 4 & 5490 & 5453 & 7 & 10 & 4 & 4989 & 4726 \\
\hline 11 & 9170 & & 11 & 3 & 4 & 5455 & 6300 & 3 & 11 & 4 & 4265 & 3987 \\
\hline 11 & 3415 & & 12 & 3 & 4 & 4031 & 4138 & 6 & & 4 & 4037 & 4256 \\
\hline 11 & 5037 & 5055 & 13 & 3 & 4 & 3704 & 3675 & 1 & 12 & 4 & 4509 & 4815 \\
\hline 12 & 4646 & 43 & 1 & 4 & 4 & 16256 & 16089 & 2 & 12 & 4 & 3263 & 3112 \\
\hline 12 & 5158 & 50 & 2 & 4 & 4 & 12010 & 12810 & 3 & 12 & 4 & 10384 & 10931 \\
\hline 113 & 5510 & 5895 & 4 & 4 & 4 & 26185 & 25967 & 2 & 14 & 4 & 4481 & 4350 \\
\hline
\end{tabular}


DESERVED ANO CALCULATED STRUCTURE FACTORS $(\times 100)$

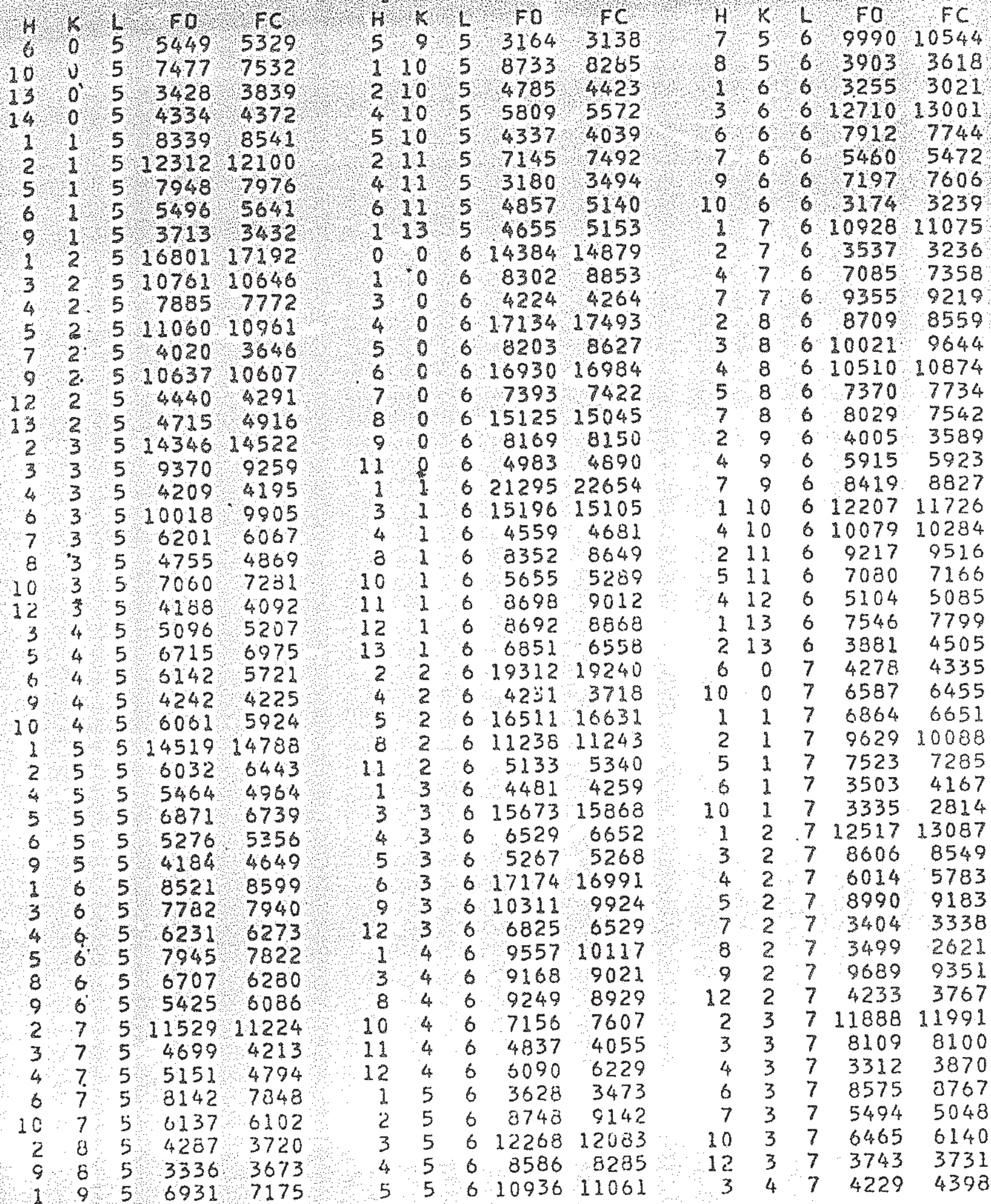




\section{OBSERVEO AND CALCULATEO STRUCTURE FACTORS $(\times 100)$}

\begin{tabular}{|c|c|c|c|c|c|c|c|c|c|c|c|}
\hline$x$ & $\mathrm{ro}$ & $\mathrm{FC}$ & $H$ & $\mathrm{~K}$ & $\mathrm{FO}$ & $6 \%$ & $H$ & K & L & $\mathrm{Fo}$ & $F C$ \\
\hline 4 & 6396 & 5512 & 2 & 2 & 4920 & 4998 & 1 & 1 & 9 & 7160 & 7273 \\
\hline 6 & 5641 & 5435 & 3 & 2 & 5260 & 5841 & 2 & 1 & 9 & 7313 & 7680 \\
\hline 4 & 4828 & 4221 & 4 & 2 & 9161 & 9248 & 5 & 1 & 9 & 5435 & 5080 \\
\hline 4 & 4773 & 5177 & 6 & 2 & 5631 & 5745 & 6 & 1 & 9 & 3356 & 3841 \\
\hline 5 & 11630 & 11695 & 9 & 2 & 6091 & 6504 & 1 & 2 & 9 & 10449 & 10975 \\
\hline 5 & 25558 & 5451 & 11 & 2 & 6318 & 5885 & 3 & 2 & 9 & 5512 & 5634 \\
\hline 5 & 3995 & 3824 & 22 & 3 & 6461 & 6370 & 4 & 2 & 9 & 6110 & 6320 \\
\hline 5 & 5993 & 5734 & 3 & 3 & 6147 & 6214 & 5 & 2 & 9 & 6929 & 7455 \\
\hline 5 & 5398 & 5122 & 7 & 3 & 5389 & 4870 & 9 & 2 & 9 & 6979 & 6976 \\
\hline 5 & 4260 & 4329 & 19 & 3 & 4352 & 3729 & 2 & 3 & 9 & 8978 & 9371 \\
\hline 6 & 8073 & 7869 & 10 & 3 & 3745 & 4472 & 3 & 3 & 8 & 6252 & 5828 \\
\hline 6 & 6999 & 6934 & 11 & 3 & 4707 & 4710 & 6 & 3 & 9 & 6288 & 5751 \\
\hline 6 & 5855 & 5758 & 1 & 4 & 810687 & 10474 & 7 & 3 & 9 & 5178 & 4344 \\
\hline 6 & 6889 & 6416 & 2 & 4 & 1848142 & 8128 & 8 & 3 & 9 & 3980 & 3750 \\
\hline 6 & 5004 & 5345 & 4 & 4 & 8318535 & 18247 & 10 & 3 & 9 & 5642 & 5500 \\
\hline 6 & 5896 & 5585 & 5 & 4 & 4296 & 3675 & 3 & 4 & 9 & 4228 & 3961 \\
\hline 7 & 8950 & 9443 & 6 & $4^{\circ}$ & 5453 & 5516 & 5 & 4 & 9 & 4361 & 4297 \\
\hline 7 & 4016 & 3697 & 7 & 4 & 812837 & 22058 & 6 & 4 & 9 & 4125 & 4485 \\
\hline 7 & 4265 & 4223 & 8 & 4 & 4106 & 13973 & 2 & 5 & 9 & 9477 & 9602 \\
\hline 7 & 3433 & 3237 & 1 & 5 & 9417 & 9676 & 2 & 5 & 9 & 5108 & 4962 \\
\hline 7 & 7417 & 7137 & 2 & 5 & 9198 & 9627 & 4 & 5 & 9 & 3501 & $348 ?$ \\
\hline 7 & 3183 & 2817 & 3 & 5 & 4760 & 4852 & 5 & 5 & 9 & 4248 & 4576 \\
\hline 9 & 6017 & 6476 & 5 & 5 & 4462 & 4325 & 1 & 6 & 9 & 482.5 & 5032 \\
\hline 10 & 6636 & 6901 & 7 & 5 & 4409 & 4439 & 3 & 6 & 9 & 5055 & 5187 \\
\hline 10 & 4202 & 3700 & 8 & 5 & 8561 & 3062 & 4 & 6 & 9 & 3842 & 4271 \\
\hline 10 & 5069 & 4775 & 9 & 5 & 4338 & 4649 & 5 & 6 & 9 & 6655 & 6590 \\
\hline $11:$ & 6036 & 6316 & 1 & 6 & 7324 & 7564 & 2 & 7 & 9 & 8301 & 8364 \\
\hline 12 & 4061 & 3668 & 2 & 6 & 3725 & 3544 & 3 & 7 & 9 & 4600 & 4131 \\
\hline 13 & 4303 & 4281 & 3 & 6 & 5114 & 4857 & 4 & 7 & 9 & 4001 & 3596 \\
\hline 0 & 829268 & 29922 & 4 & 6 & 4954 & 4701 & 6 & 7 & 9 & 6887 & 6101 \\
\hline 0 & $\approx 2 \div 5630$ & 6355 & 5 & 6 & 5529 & 5921 & 1 & 9 & 9 & 6036 & 5829 \\
\hline 0 & 815262 & 16281 & 8 & 6 & 4571 & 4404 & \& & 10 & 9 & 0220 & 6224 \\
\hline 0 & 7655 & 7989 & 1 & 7 & 5830 & 5681 & 2 & 10 & 9 & 4622 & 3817 \\
\hline 0 & 4517 & 4502 & 2 & 7 & 8213 & 8081 & 2 & 11 & 9 & 5783 & 6199 \\
\hline 0 & 6298 & 6835 & 3 & 7 & 5065 & 5069 & 0 & 0 & 10 & 5615 & 5420 \\
\hline 0 & 7434 & 7545 & 4 & 7 & 7408 & 7337 & 1 & 0 & 10 & 5902 & 5674 \\
\hline 0 & 5578 & 5846 & 5 & 7 & 3623 & 4564 & 3 & 0 & 10 & 3455 & 3698 \\
\hline 0 & 9472 & 9493 & 6 & 7 & 6260 & 5918 & 4 & 0 & 10 & 11375 & 11809 \\
\hline 2 & 453 & 4646 & 8 & 7 & 3804 & 3142 & 5 & 0 & 10 & 6934 & 6713 \\
\hline 1 & 6857 & 715 & 1 & 8 & 4168 & 3787 & 6 & 0 & 10 & 10921 & 20537 \\
\hline 1 & 4057 & 3321 & 2 & 8 & 6541 & 6876 & 7 & 0 & 10 & 6097 & 5646 \\
\hline 1 & 16258 & 16282 & 3 & 8 & 3322 & 3295 & 8 & 0 & 10 & 9757 & 9863 \\
\hline 1 & 6093 & 5508 & 4 & 8 & 4168 & 4207 & 9 & 0 & 10 & 5371 & 5414 \\
\hline 1 & 8.5665 & 579 & 6 & 8 & 4566 & 4060 & 1 & 1 & 10 & 11499 & 12970 \\
\hline 1 & $8: 17973$ & 17783 & 1 & 9 & 5729 & 5916 & 3 & 1 & 10 & 7397 & 7577 \\
\hline 1 & $8^{\circ} \quad 4127$ & 4458 & 3 & 9 & 811246 & 11439 & 8 & 1 & 10 & 6780 & 6695 \\
\hline 1 & 7831 & 704 & 5 & 9 & 5182 & 5231 & 2 & 2 & 10 & 12106 & 12134 \\
\hline 1 & 427 & 404 & $\ddot{3}$ & 11 & 3872 & 3301 & 5 & 2 & 10 & 12678 & 12707 \\
\hline 1 & 5267 & 4523 & 1 & 12 & 4208 & 3775 & 8 & 2 & 10 & 9715 & 8976 \\
\hline 2 & 8803 & 9100 & 10 & 0 & 5392 & 4965 & 3 & 3 & 10 & 9897 & 9953 \\
\hline
\end{tabular}


$\therefore$

OBSERVED AND CALCULATED STRUCTURE FACTORS $8 \times 100)$

\begin{tabular}{|c|c|c|c|c|c|c|c|c|c|c|c|c|c|c|}
\hline$k$ & $L$ & Fo & $\mathrm{FC}$ & $H$ & $k$ & L & FO & FC & $H$ & & & 5 & Fo & $\mathrm{FC}$ \\
\hline 3 & 10 & 5335 & 4844 & 1 & 11 & & 5210 & 4670 & & 0 & 8 & 2 & 4121 & 4655 \\
\hline 3 & 10 & 3911 & 3881 & 2 & 11 & & 6357 & 6345 & & 1 & 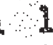 & .2 & 3406 & 3513 \\
\hline 3 & 10 & 2067 & 10701 & 5 & 11 & 1 & 4590 & 4659 & & 1 & 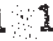 & 2 & 10254 & 9716 \\
\hline 3 & 10 & 6335 & 6647 & 1 & 21 & 1 & 7759 & 8035 & & 1 & 1 & 2 & 4039 & 3398 \\
\hline 4 & 10 & 7334 & 6991 & 3 & 21 & 1 & 4753 & 50 & 7 & 1 & 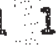 & 2 & 12447 & 11014 \\
\hline 4 & 10 & 6645 & 6347 & 4 & 2 & 1 & 3984 & 3876 & & & 2 & 2 & 5402 & 41 \\
\hline 4 & 10 & 7133 & 6984 & 5 & 2 & 1 & 6010 & 5991 & & & 2 & 2 & 3480 & 3200 \\
\hline 5 & 10 & 6810 & 6713 & 2 & 3 & 1 & 7466 & 77 & & 2 & 2 & 2 & $\$ 196$ & 4229 \\
\hline 5 & 10 & 8809 & 8753 & 3 & 3 & 1 & 5328 & 6881 & & & 2 & 2 & 5602 & 574 \\
\hline 5 & 10 & 3694 & 4650 & 6 & 3 & 1 & 5784 & 5533 & & & 2 & 2 & 4402 & 05 \\
\hline 5 & 10 & 8007 & 8800 & 5 & 4 & 1 & 3532 & 33 & & & 3 & 2 & 4188 & 4305 \\
\hline 5 & 10 & 7819 & 7549 & 1 & 5 & 1 & 6925 & 73 & & & 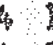 & 2 & 6556 & 6754 \\
\hline 6 & 10 & 8507 & 8410 & 5 & 5 & 1 & 4261 & 3858 & & & 4 & 2 & 11711 & 11562 \\
\hline 6 & 10 & 5875 & 5647 & 1 & 6 & 2 & 4606 & 6996 & & & 5 & 2 & 0094 & \\
\hline 7 & 10 & 766 & 81 & 5 & 6 & 1 & 4462 & 4.4 & & & 5 & 2 & 6574 & 6. \\
\hline 7 & 10 & 445 & 4701 & 2 & $7 / 1$ & 1 & 5892 & & & & 6 & 12 & 578 & \\
\hline 8 & 10 & 529 & 5864 & 1 & 91 & 1 & 4734 & 4582 & & & d: & 13 & 4516 & \\
\hline 8 & 10 & 7008 & 6774 & 0 & 0 & 2 & 19455 & 19031 & & & 2 & 13 & 5809 & \\
\hline 8 & 10 & 785 & & 2 & 01 & 2 & 4457 & 64 & & & & 13 & 5066 & \\
\hline 0 & 10 & 8472 & 8290 & 3 & 0.1 & 12 & 10358 & 10567 & & & & 13 & & \\
\hline
\end{tabular}

